\title{
Mathematical modeling of magnetic field guided colloidal particle deposition with significant electric double layer interactions
}

\author{
A. V. Alfimov, M. A. Shumova, E. M. Aryslanova, S. A. Chivilikhin \\ ITMO University, Kronverkskiy, 49, St. Petersburg, 197101, Russia \\ alfimov.anton@gmail.com
}

PACS 82.70.Dd, 75.75.Jn, 47.63.mh, 47.57.J-

DOI $10.17586 / 2220-8054-2017-8-4-426-434$

\begin{abstract}
In this work, we propose a simple theoretical method for predicting the rate and localization of magnetic field guided particle deposition from aqueous colloids. This method accounts for the colloidal electric double layer interactions between particles and vessel walls. The obtained results suggest that the colloidal interactions can be used to increase the rate of particle deposition and improve its localization.
\end{abstract}

Keywords: particle deposition, magnetic nanoparticles, magnetic particle targeting.

Received: 1 July 2017

Revised: 25 July 2017

\section{Introduction}

Colloidal solutions of magnetic particles find an increasing number of applications in biomedicine and microfluidics. This is due to the fact that the transport of these particles can be remotely controlled through application of a local magnetic field.

The biocompatibility and non-toxicity of magnetic colloids has led to their extensive use in the following: targeted drug delivery [1-6]; clinical diagnostics as contrast agents for magnetic resonance imaging [6-10]; tumor therapy by controlled hyperthermia [9-12]; cytological studies [13-17]; and in catheter embolization of blood vessels [18].

The precise control and numerous manipulation techniques for magnetic delivery enable its applications in chemical analysis and processing [19-21], lab-on-a-chip construction [21-23], and in the fabrication of functional materials [24-26].

When magnetic deposition is used for guided transport and assembly of colloidal matter, the electric double layer forces can match or even exceed the driving magnetic force. However, this type of interaction is generally omitted from theoretical studies of field guided magnetic particle transport [2,27-30].

The aim of this work is to develop a theoretical description of magnetic particle deposition that accounts for the colloidal electric double layer interactions between the particles and the vessel walls.

\section{Methods}

\subsection{Model system}

We consider an aqueous colloidal solution of spherical magnetic particles flowing through a capillary and subjected to the field of a permanent rectangular magnet (see Fig. 1A). Two coordinate systems are used throughout this work, with Cartesian coordinates $(x, y, z)$ linked to the magnet and cylindrical coordinates $(r, \theta, z)$ linked to the capillary.

The capillary is characterized by its internal radius $R$ and the closest separation $L$ between the tube interior and the magnet. The permanent magnet has the dimensions $d_{x}, d_{y}, d_{z}$ along the respective axes.

The particles have a compound structure with superparamagnetic cores embedded in a functionalized shell (see Fig. 1B). The cores transport the functional materials contained in the shell, guided by the applied magnetic field.

\subsection{Magnetic force}

The force $\mathbf{F}_{\mathbf{M}}$ acting on a particle containing superparamagnetic cores in a magnetic field $\mathbf{B}$ is given by [31]:

$$
\mathbf{F}_{\mathrm{M}}=M_{\Sigma} \nabla|\mathbf{B}|,
$$

where $M_{\Sigma}$ is the total magnetic moment of the particle cores along the applied field. The magnetic moment of an ensemble of identical superparamagnetic cores above the blocking temperature can be expressed as [32]:

$$
M_{\Sigma}=N M_{0} \Lambda\left(\frac{M_{0}|\mathbf{B}|}{k T}\right),
$$



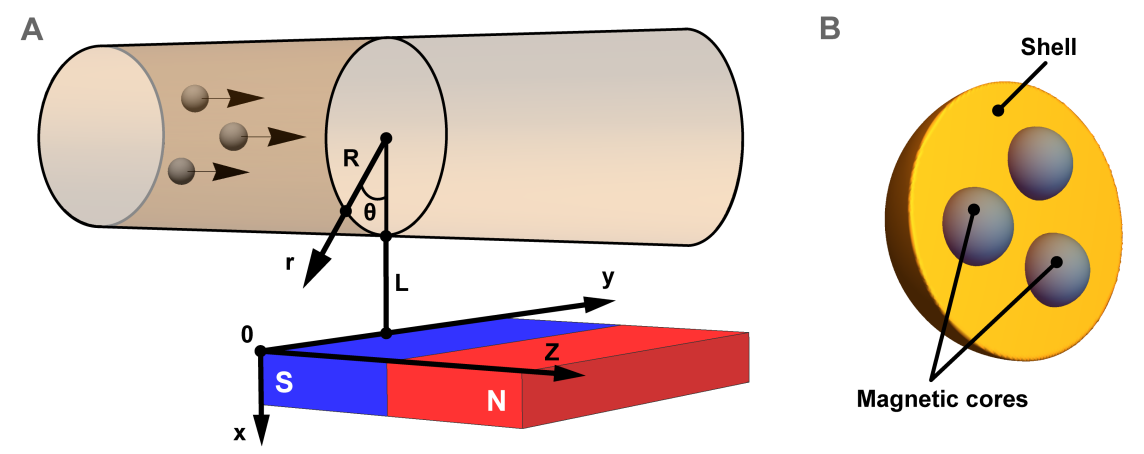

FIG. 1. A - Schematic depiction of model system. B - Model particle structure

where $\Lambda(\xi)=\operatorname{coth}(\xi)-1 / \xi$ - Langevin function; $N$ - number of magnetic cores in the particle; $M_{0}-$ maximum magnetic moment of a single core; $k$ - Boltzmann constant; $T$ - temperature. The value of $M_{0}$ can be found by fitting an experimental magnetization curve with the expression (2).

We use the analytic expressions from [33] to find the components of the magnetic field due a rectangular permanent magnet;

$$
\begin{aligned}
& B_{x}(x, y, z)= \\
& -\frac{K}{2}\left[\Gamma\left(d_{x}-x, y, z-z_{0}\right)+\Gamma\left(d_{x}-x, d_{y}-y, z-z_{0}\right)-\Gamma\left(x, y, z-z_{0}\right)-\Gamma\left(x, d_{y}-y, z-z_{0}\right)\right]_{z_{0}=0}^{z_{0}=d_{z}}, \\
& B_{y}(x, y, z)= \\
& -\frac{K}{2}\left[\Gamma\left(d_{y}-y, x, z-z_{0}\right)+\Gamma\left(d_{y}-y, d_{x}-x, z-z_{0}\right)-\Gamma\left(y, x, z-z_{0}\right)-\Gamma\left(y, d_{x}-x, z-z_{0}\right)\right]_{z_{0}=0}^{z_{0}=d_{z}}, \\
& B_{z}(x, y, z)= \\
& -K\left[\Phi\left(y, d_{x}-x, z-z_{0}\right)+\Phi\left(d_{y}-y, d_{x}-x, z-z_{0}\right)+\Phi\left(x, d_{y}-y, z-z_{0}\right)+\Phi\left(d_{x}-x, d_{y}-y, z-z_{0}\right)+\right. \\
& \left.\Phi\left(d_{y}-y, x, z-z_{0}\right)+\Phi\left(y, x, z-z_{0}\right)+\Phi\left(d_{x}-x, y, z-z_{0}\right)+\Phi\left(x, y, z-z_{0}\right)\right]_{z_{0}=0}^{z_{0}=d_{z}}
\end{aligned}
$$

where $K$ is a parameter characterizing the magnetization of the permanent magnet:

$$
\begin{gathered}
\Gamma\left(\xi_{1}, \xi_{2}, \xi_{3}\right)=\ln \left[\frac{\sqrt{\xi_{1}^{2}+\xi_{2}^{2}+\xi_{3}^{2}}-\xi_{2}}{\sqrt{\xi_{1}^{2}+\xi_{2}^{2}+\xi_{3}^{2}}+\xi_{2}}\right], \\
\Phi\left(\xi_{1}, \xi_{2}, \xi_{3}\right)= \begin{cases}\arctan \left[\frac{\xi_{1}}{\xi_{2}} \frac{\xi_{3}}{\sqrt{\xi_{1}^{2}+\xi_{2}^{2}+\xi_{3}^{2}}}\right], & y \neq 0 ; \\
0, & y=0 .\end{cases}
\end{gathered}
$$

\subsection{Colloidal force}

Solid-liquid interfaces tend to acquire charge due to surface group reactions, leading to the formation of electric double layers (EDLs) [34]. Overlapping diffuse parts of EDLs give rise to colloidal interaction forces between interfaces. For like-charged surfaces, these forces typically are repulsive [35].

The colloidal EDL interaction is completely defined by the interfacial charges and the spatial distribution of dissolved ions compensating those charges. It has been shown that the characteristic time scales of ion and particle motion allow one to approach the EDL structure problem as quasi-electrostatic [34]. For systems with low-tomedium surface potentials and absence of multivalent electrolytes in the solution, the mean-field Poisson-Boltzmann theory provides an adequate description of ion and potential distributions in the system [35].

In present work, we only consider the interactions between particles and capillary walls. The capillary radius $R$ is taken to be much larger than the particle radius $a$ and the characteristic charge-screening distance in the solution 
(Debye length is less than $1 \mu \mathrm{m}$ in aqueous solutions). Therefore, we can use the Poisson-Boltzmann equation solution for a flat wall to describe the electric potential distribution $\phi(r)$ in capillary's EDL [35]:

$$
\phi(r)=2 \frac{k T}{e} \ln \left(\frac{1+\lambda \exp [-\kappa(R-r)]}{1-\lambda \exp [-\kappa(R-r)]}\right), \quad \lambda=\tanh \left(\frac{e \zeta_{\mathrm{C}}}{4 k T}\right),
$$

where $\zeta_{\mathrm{C}}$ - surface (zeta) potential of capillary walls; $\kappa$ - inverse Debye screening length:

$$
\kappa^{2}=\frac{2 I e^{2}}{\varepsilon \varepsilon_{0} k T}
$$

$I$ - ionic strength of the solution; $\varepsilon$ - dielectric permittivity of the solution; $\varepsilon_{0}$ - dielectric constant.

The dominant component of the colloidal EDL interaction is given by the electrostatic force exerted on the particles by the charged capillary wall and the ions of its EDL. This force can be found from (6) as [35]:

$$
\mathbf{F}_{\mathrm{C}}=\mathbf{n}_{\mathrm{W}} Q_{\mathrm{eff}} 4 \frac{k T}{e} \frac{\kappa \lambda \exp [-\kappa(R-r)]}{1-(\lambda \exp [-\kappa(R-r)])^{2}},
$$

where $\mathbf{n}_{\mathrm{W}}=-\mathbf{r} /|\mathbf{r}|$ - unit vector normal to the capillary wall, pointing inwards; $Q_{\mathrm{eff}}-$ effective particle charge that can be related to particle surface (zeta) potential $\zeta_{\mathrm{P}}$ as $[36]$ :

$$
Q_{\mathrm{eff}}=4 \pi \varepsilon \varepsilon_{0} \zeta_{\mathrm{P}} a \exp (\kappa a) .
$$

\subsection{Magnetic particle deposition}

We now study the motion of magnetic particles in the described system. The particles are considered to be carried through the capillary by a laminar liquid flow exhibiting Poiseuille velocity profile. Only the most significant forces acting on the particles are considered: the magnetic force, the viscous drag and the colloidal EDL interaction with capillary walls. The resulting equations of motion for a magnetic colloidal particle are:

$$
\begin{aligned}
& \dot{\mathbf{V}}=\frac{\mathbf{F}_{\mathbf{M}}+\mathbf{F}_{\mathrm{C}}}{m}-\gamma\left(\mathbf{V}-\mathbf{V}_{\mathrm{P}}\right), \\
& \left.\mathbf{V}\right|_{t=0}=\mathbf{V}_{0},
\end{aligned}
$$

where $\mathbf{V}$ - particle velocity; $\mathbf{F}_{\mathbf{M}}$ - magnetic force (1); $\mathbf{F}_{\mathbf{C}}$ - colloidal force (7); $m$ - total mass of the particle; $\gamma=6 \pi \eta a / m$ - viscous friction coefficient; $\eta$ - dynamic viscosity of the liquid; $\mathbf{V}_{\mathbf{P}}$ - liquid flow velocity given by:

$$
\mathbf{V}_{\mathbf{P}}=\mathbf{i}_{z} V_{\mathrm{P}}^{\max }\left(1-\frac{r^{2}}{R^{2}}\right)
$$

here $\mathbf{i}_{z}$ - unit vector in the direction of $z$ axis; $V_{\mathrm{P}}^{\max }$ - maximum flow velocity.

When the inertial term $\dot{\mathbf{V}}$ in (8) is negligible, the system evolves in a quasi-stationary regime (QSR). The velocity a particle would have in QSR is denoted as $\mathbf{U}$ and can be found from (8) as:

$$
\mathbf{U}=\frac{\mathbf{F}_{\mathbf{M}}+\mathbf{F}_{\mathrm{C}}}{\gamma m}+\mathbf{V}_{\mathrm{P}}
$$

We will now derive a sufficient a priori condition for the quasi-stationary regime in the considered system.

The solution to the original system (8) has the following form:

$$
\mathbf{V}=\mathbf{V}_{0} e^{-\gamma t}+\int_{0}^{t} e^{-\gamma\left(t-t^{\prime}\right)}\left(\frac{\mathbf{F}_{\mathbf{M}}+\mathbf{F}_{\mathbf{C}}}{m}+\gamma \mathbf{V}_{\mathbf{P}}\right) \mathrm{d} t^{\prime}
$$

We note that by using the definition (10), the solution (11) can be rewritten as:

$$
\mathbf{V}=\mathbf{V}_{0} e^{-\gamma t}+\gamma \int_{0}^{t} e^{-\gamma\left(t-t^{\prime}\right)} \mathbf{U}\left(t^{\prime}\right) \mathrm{d} t^{\prime}
$$

Expression (12) describes a system with memory of its previous states, and the memory function is decaying exponentially. The memory span of this system can be estimated as $\Delta t=3 / \gamma$, corresponding to a tenfold decay of the exponential function.

Therefore, the particle velocity when $t>\Delta t$ can be found as:

$$
\mathbf{V}=\gamma \int_{t-\Delta t}^{t} e^{-\gamma\left(t-t^{\prime}\right)} \mathbf{U}\left(t^{\prime}\right) \mathrm{d} t^{\prime}
$$


From the first mean value theorem for definite integrals, there is such $\tilde{t}_{i}, t-\Delta t<\tilde{t}_{i}<t$, that:

$$
V_{i}(t)=U_{i}\left(\tilde{t}_{i}\right) \gamma \int_{t-\Delta t}^{t} e^{-\gamma\left(t-t^{\prime}\right)} \mathrm{d} t^{\prime}=U_{i}\left(\tilde{t}_{i}\right),
$$

where $i=x, y, z$ - coordinate index; $V_{i}, U_{i}-i$-th component of particle velocity vector and quasi-stationary velocity vector respectively.

According to (14), when the QSR velocity $U_{i}$ varies insignificantly over the time $\Delta t$ so that $U_{i}\left(\tilde{t}_{i}\right) \approx U_{i}(t)$, the quasi-stationary regime ensues. Hence, we can formulate the sufficient condition for the QSR:

$$
\left|\frac{U_{i}(t)-U_{i}(t-\Delta t)}{U_{i}(t)}\right| \ll 1 .
$$

This condition, however, requires the prior knowledge of particle trajectories and cannot be checked $a$ priori.

We now assume that the quasi-stationary velocity $U_{i}$ varies linearly with time over the course of $\Delta t$ :

$$
U_{i}(t)-U_{i}(t-\Delta t)=\left.\dot{U}_{i}\right|_{t} \Delta t
$$

Then, the condition (15) reduces to:

$$
\Delta t\left|\frac{\left.\dot{U}_{i}\right|_{t}}{U_{i}(t)}\right| \ll 1
$$

We note that when the forces acting on the particle and the liquid flow velocity profile do not change in time, the following is true:

$$
\dot{U}_{i}=\left(\mathbf{V} \cdot \nabla U_{i}\right)
$$

From the definition of $\tilde{t}_{i}$, it follows that:

$$
t-\tilde{t}_{i}=\alpha_{i} \Delta t, \quad 0<\alpha_{i}<1
$$

and, using (14):

$$
V_{i}(t)=U_{i}\left(\tilde{t}_{i}\right)=U_{i}(t)-\left.\dot{U}_{i}\right|_{t} \alpha_{i} \Delta t
$$

If the condition (16) is satisfied for all coordinates:

$$
\left|U_{i}\right| \gg \Delta t\left|\dot{U}_{i}\right|, \quad \forall i, \quad i=x, y, z,
$$

then, according to (18):

$$
\left|U_{i}\right| \gg \alpha_{i} \Delta t\left|\dot{U}_{i}\right|, \quad \forall i, \quad i=x, y, z .
$$

Thus, combining (17), (19) and (20), we can finally rewrite the sufficient condition for quasi-stationary regime (16) as:

$$
\Delta t\left|\frac{\left(\mathbf{U} \cdot \nabla U_{i}\right)}{U_{i}}\right| \ll 1, \quad \forall i, \quad i=x, y, z .
$$

The resulting expression (21) can be directly evaluated at each point of the considered system and does not require the prior knowledge of particle trajectories. Therefore, (21) is the sufficient a priori condition for the quasi-stationary regime.

In areas where condition (21) is met, the magnetic particle velocity field can be obtained directly from (10).

To evaluate the rate and the localization of the particle deposition in an external magnetic field, we use the obtained velocity field to calculate the particle flux onto the capillary walls. From the continuity equation for a steady flow $\nabla \cdot(C \mathbf{U})=0$, we get the following expression for the particle flux $j_{\mathrm{P}}$ :

$$
j_{\mathrm{P}}=\left(\mathbf{n}_{\mathrm{W}} \cdot \mathbf{U}\right) C_{0} \exp \left[-\int_{0}^{t}(\nabla \cdot \mathbf{U}) \mathrm{d} t^{\prime}\right],
$$

where $C$ - local particle concentration; $C_{0}$ - particle concentration far from the magnet. 


\section{Results and discussion}

We presented a simple theoretical method for predicting the particle flux during the magnetic deposition from colloids that accounts for the EDL interactions between particles and capillary walls. The method is realized in three steps:

(1) Evaluation of the quasi-stationary regime condition (21) for the considered system to check for the method applicability.

(2) Analytical calculation of the particle velocity field from (10).

(3) Numerical computation of the particle flux onto the capillary walls using (22).

Note that for the rough estimate of particle flux, the local particle concentration can be equated to the bulk concentration $C_{0}$, resulting in analytical expression for the flux: $j_{\mathrm{P}}=\left(\mathbf{n}_{\mathrm{W}} \cdot \mathbf{U}\right) C_{0}$.

Condition (21) poses limits on the spatial rate of change for forces acting on the particle and on the maximum velocity of the flow. For colloidal interactions, this generally means that the method is applicable for systems with either very high ionic strength (so that the EDL forces are rendered insignificant), or low ionic strength (less than $\left.10^{-5} \mathrm{M}\right)$.

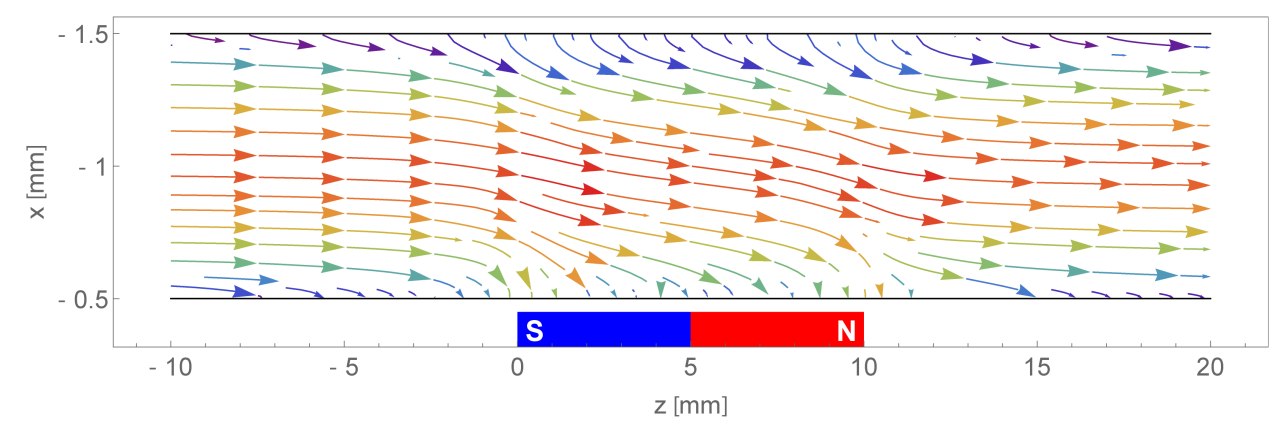

FIG. 2. Streamlines of particle velocity field in the capillary central cross section perpendicular to the surface of the magnet and the direction of liquid flow. Inner capillary radius $R=0.5 \mathrm{~mm}$; distance from the magnet $L=0.5 \mathrm{~mm}$ (depicted not to scale); particles with radius of $a=600 \mathrm{~nm}$ are submerged in water at room temperature flowing with maximum velocity of $\mathrm{V}_{P}^{\max }=0.2 \mathrm{~m} / \mathrm{s}$; Reynolds number $R e=10^{2}$

We now demonstrate how the suggested method can be used to study the effects of system parameters on magnetic particle deposition.

Properties of the magnetic cores are chosen to reflect those of the $10 \mathrm{~nm}$ magnetite nanoparticles reported in [37], with maximum magnetic moment of a single core $M_{0}=178 \cdot 10^{-21} \mathrm{~N} \cdot \mathrm{m} / \mathrm{T}$. The bulk particle concentration is fixed at $C_{0}=10^{15} 1 / \mathrm{m}^{3}$.

With the functional material located in the particle shell, we take the shell volume to be the same for all considered particle sizes and equal to $0.065 \mu \mathrm{m}^{3}$. Because of that, the shell material mass flux is always directly proportional to the particle flux demonstrated in the following sections.

For simplicity, in this work we consider the surface potentials of the capillary and the particles to be equal: $\zeta_{\mathrm{C}}=\zeta_{\mathrm{P}}=\zeta$.

The parameters of the magnet are based on the rectangular neodymium magnet from [37], with dimensions of $d_{x}=30 \mathrm{~mm}, d_{y}=20 \mathrm{~mm}, d_{z}=10 \mathrm{~mm}$ and magnetization constant $K=0.269 \mathrm{~N} / \mathrm{A} \cdot \mathrm{m}^{2}$.

A typical particle velocity field for such systems is depicted in Fig. 2.

\subsection{Particle flux in a system imitating a blood vessel}

First, we consider a model system with parameters matching those of a human artery. The liquid is at body temperature and has the viscosity and the ionic strength of blood $(\eta=2.8 \mathrm{mPa} \cdot \mathrm{s}$ and $I=154 \mathrm{mM})$. At high ionic strength values for this system, the EDL interactions are usually insignificant [35]. Maximum flow velocity is $V_{\mathrm{P}}^{\max }=0.9 \mathrm{~m} / \mathrm{s}$, inner vessel radius is $R=2 \mathrm{~mm}$, Reynolds number is $R e=6 \cdot 10^{2}$.

The deposition of $3 \mu \mathrm{m}$ magnetic particles under the applied magnetic field is studied for three different separations between the vessel interior and the magnet (see Fig. 3). These correspond to different blood vessel depths. As demonstrated in Fig. 3, the suggested method allows prediction of both the rate and localization of particle deposition for the considered systems. As the magnetic force decays further away from the magnet, the particles flux decreases and becomes less localized. 

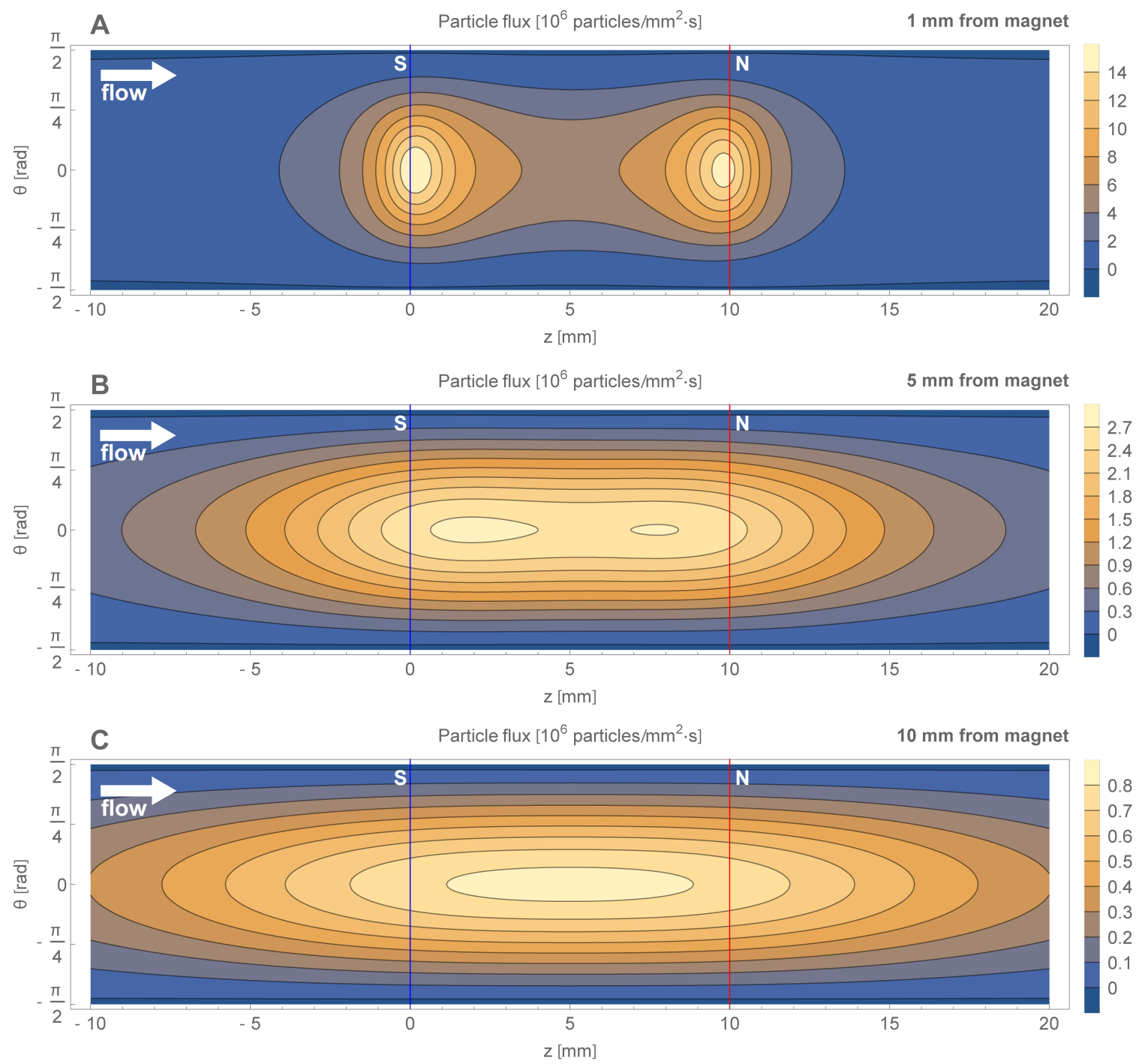

FIG. 3. Particle flux distribution over the capillary wall in a system imitating human artery with the vessel interior separated from the magnet by: $\mathrm{A}-1 \mathrm{~mm}, \mathrm{~B}-5 \mathrm{~mm}, \mathrm{C}-10 \mathrm{~mm}$. Lines indicate the location of magnetic poles beneath the capillary

For a given vessel depth, the particle flux can be adjusted by changing the volume of magnetic cores embedded in the particle. This is illustrated in Fig. 4.

\subsection{Particle flux in a microfluidic system at low ionic strength}

Second, we consider a system with magnetic particles carried by an aqueous solution with low ionic strength. In this case, the EDL interaction between the particles and the capillary walls can be significant, depending on their surface potentials.

The liquid is at room temperature and has an ionic strength of $I=10^{-3} \mathrm{mM}$. Maximum flow velocity is $V_{\mathrm{P}}^{\max }=0.5 \mathrm{~m} / \mathrm{s}$, capillary interior is separated from the magnet by $L=1 \mathrm{~mm}$ and has the radius of $R=0.5 \mathrm{~mm}$; Reynolds number is $R e=3 \cdot 10^{2}$.

The magnetic deposition of $700 \mathrm{~nm}$ particles is compared for different values of surface potentials in the system (see Fig. 5). When a non-negligible interfacial charge is present, a repulsion counteracting the magnetic force arises. As a result, with increasing surface potential the area of particle deposition localization decreases (see Fig. 5B,C) until the particle flux onto the capillary wall is terminated.

At the same time, colloidal EDL forces slow down the approaching particles and focus them around the magnet poles, thus increasing the local particle concentration. This may result in a higher particle flux when compared to a system with no colloidal repulsion, as seen in Fig. 5A,B. Further rise in surface potential, however, will lead to 


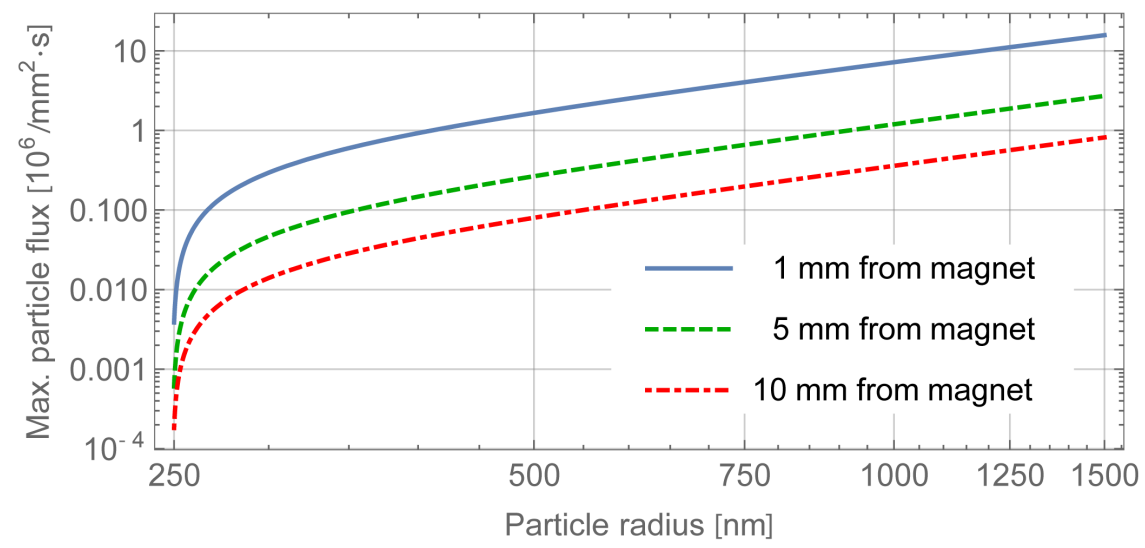

FIG. 4. Maximum particle flux as a function of particle size (with shell volume being fixed) at different separations between vessel interior and the magnet
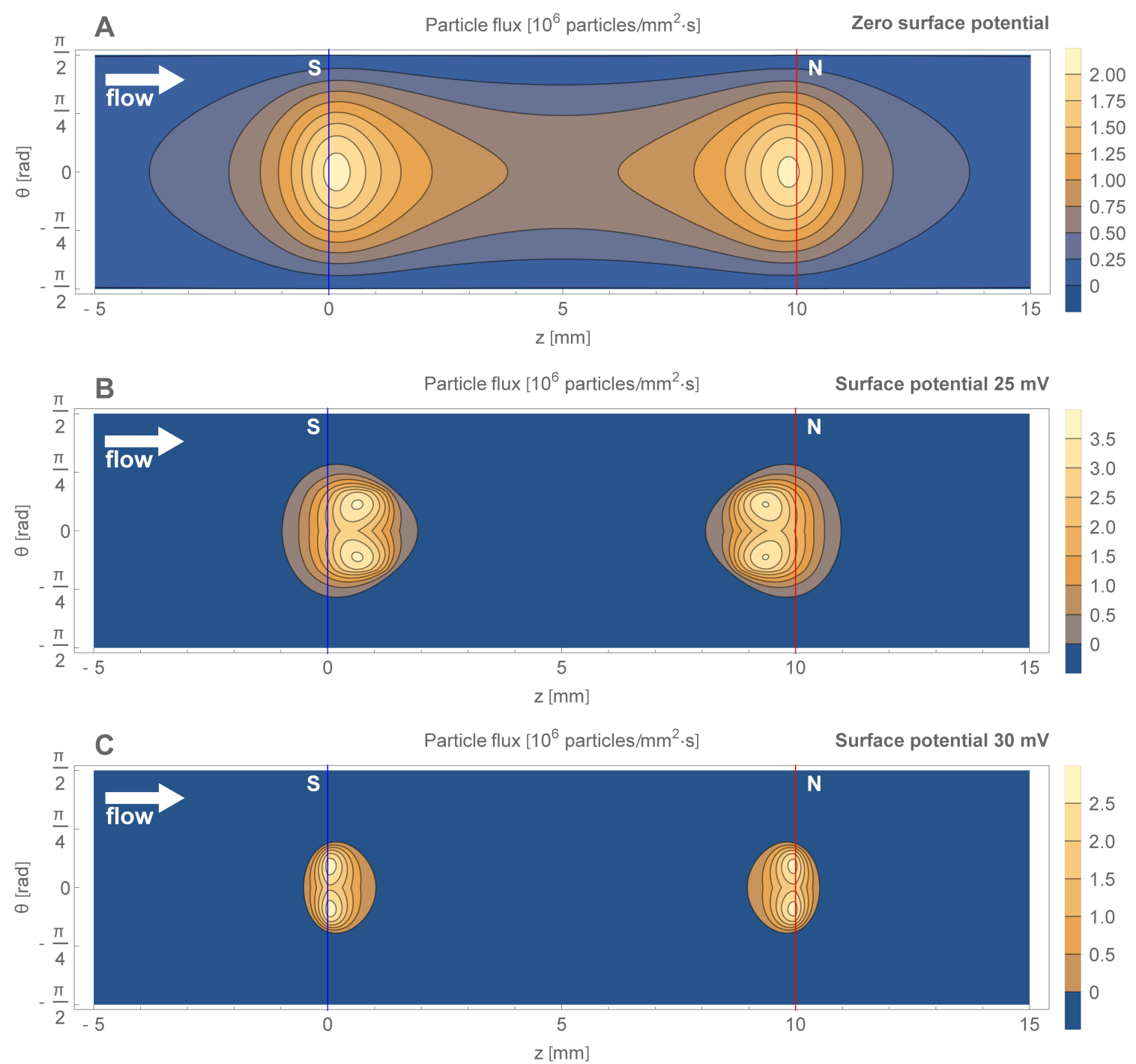

FIG. 5. Particle flux distribution over the capillary wall in systems exhibiting different surface potential: $\mathrm{A}-0, \mathrm{~B}-25 \mathrm{mV}, \mathrm{C}-30 \mathrm{mV}$. Lines indicate the location of magnetic poles beneath the capillary 
particle flux decrease (compare Fig. 5B,C). This is due to particle velocity drop overpowering the gain in local concentration. The balancing between particle concentration and velocity near capillary wall also explains the bifurcation of the flux maxima observed in Fig. 5.

\section{Conclusions}

The work proposes a simple theoretical method for predicting the rate and localization of magnetic particle deposition from aqueous colloids. This method accounts for electric double layer interactions between particles and capillary walls in low ionic strength solutions. When applicable, the method allows one to estimate the system parameters required for achieving a specified deposition regime.

The results obtained for the magnetic deposition with significant electric double layer forces suggest that the presence of colloidal interactions can increase the rate of particle deposition and improve its localization.

\section{References}

[1] Al-Jamal K.T., Bai J., Wang J.T.-W., Protti A., Southern P., Bogart L., Heidari H., Li X., Cakebread A., Asker D., Al-Jamal W.T., Shah A., Bals S., Sosabowski J., Pankhurst Q.A. Magnetic Drug Targeting: Preclinical in Vivo Studies, Mathematical Modeling, and Extrapolation to Humans. Nano Lett., 2016, 16, P. 5652-5660.

[2] Sharma S., Katiyar V.K., Singh U. Mathematical modelling for trajectories of magnetic nanoparticles in a blood vessel under magnetic field. Journal of Magnetism and Magnetic Materials, 2015, 379, P. 102-107.

[3] Kulkarni S., Ramaswamy B., Horton E., Gangapuram S., Nacev A., Depireux D., Shimoji M., Shapiro B. Quantifying the motion of magnetic particles in excised tissue. Effect of particle properties and applied magnetic field. Journal of Magnetism and Magnetic Materials, 2015, 393, P. 243-252.

[4] Shapiro B., Kulkarni S., Nacev A., Muro S., Stepanov P.Y., Weinberg I.N. Open challenges in magnetic drug targeting. WIREs Nanomed. Nanobiotechnol., 2014, 7, P. 446-457.

[5] Shapiro B., Kulkarni S., Nacev A., Sarwar A., Preciado D., Depireux D.A. Shaping Magnetic Fields to Direct Therapy to Ears and Eyes. Annu. Rev. Biomed. Eng., 2014, 16, P. 455-481.

[6] Li X., Wei J., Aifantis K.E., Fan Y., Feng Q., Cui F.-Z., Watari F. Current investigations into magnetic nanoparticles for biomedical applications. J. Biomed. Mater. Res., 2016, 104, P. 1285-1296.

[7] Bonnemain B. Superparamagnetic agents in magnetic resonance imaging: physiochemical characteristics and clinical applications-a review. J. Drug Target, 1998, 6, P. 167-174.

[8] Weissleder, R., Bogdanov, A., Neuwelt, E.A., Papisov, M. Long circulating iron oxides for MR imaging. Adv. Drug. Delivery Rev., 1995, 16, P. 321-334.

[9] Berry C.C., Curtis A.S.G. Functionalisation of magnetic nanoparticles for applications in biomedicine. J. Phys. D. Appl. Phys., 2003, 36, R198-R206.

[10] Harisinghani M.G., Barentsz J., Hahn P.F., Deserno W. M., Tabatabaei S., van de Kaa C. H., de la Rosette J., Weissleder R. Noninvasive detection of clinically occult lymph-node metastases in prostate cancer. N. Engl. J. Med., 2003, 348, P. $2491-2499$.

[11] Jordan A., Wust P., Fähling H., John W., Hinz A., Felix R. Inductive heating of ferromagnetic particles and magnetic fluids: physical evaluation of their potential for hyperthermia. Int. J. Hyerthermia, 1997, 9, P. 51-68.

[12] Neilsen O.S., Horsman M., Overgaard J. A future hyperthermia in cancer treatment. E. J. Cancer, 2001, 37, P. 1587-1589.

[13] Tibbe A.G., de Grooth B.G., Greve J., Liberti P.A., Dolan G.J., Terstappen L.W. Optical tracking and detection of immunomagnetically selected and aligned cells. Nature Biotechnol., 1999, 17, P. 1210-1213.

[14] Kularatne B.Y., Lorigan P., Browne S., Suvarna S.K., Smith M.O., Lawry J. Monitoring tumour cells in the peripheral blood of small cell lung cancer patients. Cytometry, 2002, 50, P. 160-167.

[15] Zigeuner R.E., Riesenberg R., Pohla H., Hofstetter A., Oberneder R. Isolation of circulating cancer cells from whole blood by immunomagnetic cell enrichment and unenriched immunocytochemistry in vitro. J. Urol., 2003, 169, P. 701-705.

[16] Morisada S., Miyata N., Iwahori K. Immunomagnetic separation of scum-forming bacteria using polyclonal antibody that recognizes mycolic acids. J. Microbiol. Methods, 2002, 51, P. 141-148.

[17] Tan W., Wang K., He X., Zhao X.J., Drake T., Wang L., Bagwe R.P. Bionanotechnology based on silica nanoparticles. Medicinal Research Reviews, 2004,24(5), P. 621-638.

[18] Korolev D.V., Naumisheva E.B., Gareev K.G., Luchinin V.V., Panov M.F., Permyakov N.V. Colloidal particles based on silica coated iron oxide for MRI-controlled catheter embolization. Translational Medicine, 2015, 2(4), P. 48-53.

[19] Shukla N., Saxena A., Gupta V., Rawat A.S., Kumar V., Shrivastava S., Rajagopal C., Rai P.K. Magnetic silica nanoparticles for the removal of $\mathrm{Pb}^{+2}$ from water. Nanosystems: Physics, Chemistry, Mathematics, 2016, 7(3), P. 488-491.

[20] Gijs M.A.M., Lacharme F., Lehmann U. Microfluidic Applications of Magnetic Particles for Biological Analysis and Catalysis. Chem. Rev., 2010, 110, P. 1518-1563.

[21] Jamshaid T., Neto E.T.T., Eissa M.M., Zine N., Kunita M.H., El-Salhi A.E. Elaissari A. Magnetic particles: From preparation to lab-on-achip, biosensors, microsystems and microfluidics applications. Past, Present and Future challenges of Biosensors and Bioanalytical tools in Analytical Chemistry: a tribute to Prof Marco Mascini, 2016, 79, P. 344-362.

[22] Sajeesh P., Sen A.K. Particle separation and sorting in microfluidic devices: a review. Microfluidics and Nanofluidics, 2014,17 , P. 1-52.

[23] Cao Q., Han X., Li L. Configurations and control of magnetic fields for manipulating magnetic particles in microfluidic applications: magnet systems and manipulation mechanisms. Lab Chip, 2014, 14, P. 2762-2777.

[24] Ortega García B., Kharissova O.V., Rasika Dias H.V., Servando Aguirre T. F., Salinas Hernández J. Nanocomposites with antibacterial properties using CNTs with magnetic nanoparticles. Nanosystems: Physics, Chemistry, Mathematics, 2016, 7(1), P. 161-168.

[25] Wang M., Le He, Yin Y. Magnetic field guided colloidal assembly. Materials Today, 2013, 16, P. $110-116$. 
[26] Chen C.-H., Abate A.R., Lee D., Terentjev E.M. Weitz D.A. Microfluidic Assembly of Magnetic Hydrogel Particles with Uniformly Anisotropic Structure. Adv. Mater. , 2009, 21, P. 3201-3204.

[27] Bernad S.I., Totorean A.F., Vekas L. Particles deposition induced by the magnetic field in the coronary bypass graft model. Journal of Magnetism and Magnetic Materials, 2016, 401, P. 269-286.

[28] Larimi M.M., Ramiar A., Ranjbar A.A. Numerical simulation of magnetic nanoparticles targeting in a bifurcation vessel. Journal of Magnetism and Magnetic Materials, 2014, 362, P. 58-71.

[29] Heidsieck A., Vosen S., Zimmermann K., Wenzel D., Gleich B. Analysis of Trajectories for Targeting of Magnetic Nanoparticles in Blood Vessels. Mol. Pharmaceutics, 2012, 9, P. 2029-2038.

[30] Gitter K., Odenbach S. Investigations on a Branched Tube Model in Magnetic Drug Targeting - Systematic Measurements and Simulation. IEEE Trans. Magn., 2012, 49, P. 343-348.

[31] Kyrilenko A.V., Chekhun V.F., Podoltsev A.D., Kondratenko I.P., Kucheryavaya I.N., Bondar V.V., Shpilevaya S.I., Todor I.N. Analysis of the force action of a high-gradient magnetic field on magnetic nanoparticles in a flowing fluid. Reports of the National Academy of Sciences of Ukraine, 2010, 9, P. 162-172.

[32] Kronmüller H., Parkin S. Handbook of Magnetism and Advanced Magnetic Materials. John Wiley \& Sons, 2007,3064 pp.

[33] Xiao-fan G., Yong Y., Xiao-jing Z. Analytic expression of magnetic field distribution of rectangular permanent magnets, Applied Mathematics and Mechanics, 2004, 25(3), P. 297-306.

[34] Lyklema J. Fundamentals of Interface and Colloid Science. Volume II: Solid-Liquid Interfaces. Academic Press, 2005,787 pp.

[35] Lyklema J. Fundamentals of Interface and Colloid Science. Volume IV: Particulate colloids. Academic Press, 2005,692 pp.

[36] Alfimov A.V., Aryslanova E.M., Chivilikhin S.A. An analytical method for determining the interaction energy between multiple identical spherical colloidal zinc oxide nanoparticles. J. Phys.: Conf. Ser., 2014, 541, P. 012063.

[37] Korolev D.V., Galagudza M.M., Afonin M.V., Shutkevitch V.V. Magnetic field-guided delivery of magnetite nanoparticles in the model in vitro system. Translational Medicine, 2015, 2(4), P. 20-27. 